\title{
Automated image acquisition and analysis of beam sensitive samples
}

\author{
Eric Stach ${ }^{1}$, Dmitri N. Zakharov, ${ }^{1}$ Yuewei Lin, ${ }^{2}$ Shinjae Yoo, ${ }^{2}$ and Guenter Resch $^{3}$ \\ 1. Center for Functional Nanomaterials, Brookhaven National Laboratory, Upton, USA. \\ 2. Computational Sciences Initiative, Brookhaven National Laboratory, Upton, USA. \\ 3. Nexperion, e.U., Vienna, Austria.
}

The development of direct electron detector technology [1,2] has led to a revolution in the field of cryo electron microscopy, [3] leading to dramatic enhancements in image resolution. [4] These detectors provide a greatly enhanced detective quantum efficiency, which can lead to both high image acquisition speeds and operation in low-dose modes wherein every electron that strikes the detector is counted ("counting mode").

In this presentation, we report on our efforts to take full advantage of this novel detector to image beam sensitive 'solid state' materials (as opposed to biological materials). In the case of phase contrast imaging of solid state materials, it is necessary to have the sample oriented along a zone axis orientation. However, for many beam sensitive materials it is not possible to effectively tilt the sample as this requires electron beam exposure. This is further exacerbated in the case of nanomaterials, where conventional sample preparation approaches based on drop-casting led to the deposition of crystals in random orientation with respect to the substrate / electron beam.

The approach we have taken relies upon the integration of several different elements of the microscope. We are utilizing a G1 Titan 80/300 TEM equipped with a post-specimen corrector for phase contrast imaging. The instrument is also equipped with a Gatan K2-IS Direct Electron Detector [2], running Digital Micrograph GMS3. We have installed the SerialEM [5] software package to allow control over the microscope, thereby enabling automated image acquisition. The intent is to automatically acquire many images (similar to the approach used in single particle methods in biology), and use automated image analysis methods to identify images wherein the crystals are well oriented. As of the time of the writing of this abstract, we have just completed all necessary software installations and calibrations.

The talk will focus on the motivation, software integration approach, the methodology appropriate for automated image acquisition and the analysis techniques used to select aligned images from the generated data stream. Examples from the imaging of zeolites, 1D and 2D metal organic framework structures and variety of semi-crystalline polymers will be described.

\section{References:}

[1] Battaglia M, Contarato D, Denes P, Doering D, Giubilato P, Kim T S, Mattiazzo S, Radmilovic V and Zalusky S, Nucl Instrum Meth A 598 2009, pp. 642-9

[2] http://www.gatan.com/products/tem-imaging-spectroscopy/k2-camera

[3] Callaway, E., 2015, September 9, The revolution will not be crystallized: a new method sweeps through structural biology, Retrieved from www.nature.com

[4] Bartesaghi A, Merk A, Banerjee S, Matthies D, Wu X, Milne JLS, and Subramaniam S, Science. 348, 2015, pp. 1147-1151.

[5] http://bio3d.colorado.edu/SerialEM/ 
[6] This research used resources of the Center for Functional Nanomaterials, which is a U.S. DOE Office of Science Facility, at Brookhaven National Laboratory under Contract No. DE-SC0012704 